\title{
Fevicacia clínica de la aspiración supra glótica a través del uso del
Nuevo Dispositivo de Invención SUPRAtube en Pacientes Intubados y
Ventilados Mecánicamente.
}

Ramírez-Sarmiento A, Reyes C, López N, Pedroso K, Cáceres-Rivera D, Espitia A, Rey D, Bermon A, Pizarro C, Orozco-Levi M. Grupo EMICON. Hospital Internacional de Colombia. Fundación Cardiovascular de Colombia. Floridablanca, Santander, Colombia

INTRODUCCION Y OBJETIVO

Uno de los factores de riesgo para la neumonía asociada a la ventilación es la broncoaspiración intermitente o continua del contenido orofaríngeo. Nuestro grupo ha diseñado y construido un dispositivo de aspiración supraglótica continua que puede disminuir la broncoaspiración y sus complicaciones. Objetivo: Evaluar la eficacia del SUPRAtube para la aspiración continua en pacientes intubados y ventilados mecánicamente, y su potencial beneficio clínico en la prevención de riesgo de complicaciones.

\begin{tabular}{|c|c|c|c|c|c|}
\hline \multicolumn{6}{|l|}{\begin{tabular}{|l} 
Comorbilidades \\
$\mathrm{n}(\%)$
\end{tabular}} \\
\hline Infección VAS & 43 & $1(2)$ & $0(0)$ & $1(4)$ & 1,000 \\
\hline Dialisis & 48 & $11(23)$ & $5(29)$ & $6(19)$ & 0,486 \\
\hline ECMO & 48 & $1(2)$ & $0(0)$ & $1(3)$ & 1,000 \\
\hline Sepsis & 43 & $15(35)$ & $7(44)$ & $8(30)$ & 0,509 \\
\hline Ninguna & 42 & $23(55)$ & $8(50)$ & $15(58)$ & 0,753 \\
\hline \multicolumn{6}{|l|}{$\begin{array}{l}\text { Secreciones } \\
\text { n(\%) }\end{array}$} \\
\hline Purulentas & 45 & $20(44)$ & $6(35)$ & $14(50)$ & 0,372 \\
\hline Mucoides & 44 & $17(39)$ & $8(47)$ & $9(33)$ & 0,526 \\
\hline Mucopurulentas & 44 & $5(11)$ & $3(18)$ & $2(61)$ & 0,359 \\
\hline Sanguinolentas & 44 & $5(11)$ & $2(12)$ & $3(11)$ & 1,000 \\
\hline $\begin{array}{l}\text { Secreciores Orofaríngesas } \\
\mathrm{n}(\%)\end{array}$ & 43 & $1(2)$ & $1(6)$ & $0(0)$ & 0.372 \\
\hline $\begin{array}{l}\text { Medicamentos que Aumenten o } \\
\text { disminuyan las secreciones } \\
\text { Orofaríngeas } \\
\mathrm{n}(\%)\end{array}$ & 44 & $1(2)$ & $1(6)$ & $0(0)$ & 0,364 \\
\hline \multicolumn{6}{|l|}{$\begin{array}{l}\text { Antecedentes } \\
\mathrm{n}(\%)\end{array}$} \\
\hline Patológicos & 44 & $33(75)$ & $11(73,3)$ & $22(76)$ & 1,0000 \\
\hline Farmalógico & 44 & $20(45)$ & $9(60)$ & $11(38)$ & 0,210 \\
\hline Qx Abdominal/Otras & 44 & $9(20)$ & $3(20)$ & $6(21)$ & 1,000 \\
\hline Qx Torax & 43 & $41(95,3)$ & $15(100)$ & $26(92,9)$ & 0,535 \\
\hline Qx Cervical & 43 & $1(2)$ & $0(0)$ & $1(3)$ & 1,000 \\
\hline Toxicológicos & 44 & $13(30)$ & $5(33)$ & $8(28)$ & 0,737 \\
\hline Exposicionales & 45 & $9(20)$ & $5(31)$ & $4(14)$ & 0,245 \\
\hline
\end{tabular}

\section{MATERIALES Y MÉTODOS}

Ensayo clínico aleatorizado que evalúa la eficacia del SUPRAtube vs el tratamiento convencional. Se han incluido $n=50$ pacientes adultos (edad, $56 \pm 19$ años) intubados y ventilados

mecánicamente. El dispositivo fue ubicado en supraglotis por vía bucal en las primeras 12 horas post-intubación

por videonasofibrolaringoscopia iniciual en la cual se realizó la toma de secreciones y estudios microbiológicos ampliados. El dispositivo se conectó a presión negativa continua (-20 a -30 $\mathrm{cmH2O}$ ). Se repitieron los estudios endoscópicos inmediatamente antes de la extubación.

\begin{tabular}{|c|c|c|c|c|c|}
\hline \multicolumn{6}{|c|}{ Evaluación de la Eficacia de Aspiración del SUPRAtube } \\
\hline \multicolumn{6}{|c|}{ EFICACIA SUPRAtube $n(\%)$} \\
\hline & $\mathrm{n}$ & Total & $\begin{array}{l}\text { Tratamiento } \\
\text { Estandar }\end{array}$ & SUPRAtube & p Valor \\
\hline $\begin{array}{l}\text { Volumen Aspiración Final } \\
\text { Media (SD) }\end{array}$ & 43 & $172(227)$ & $27(28)$ & $258(249)$ & $0,000^{*}$ \\
\hline Peso Aspirado Final & 42 & $382(339)$ & $208(189)$ & $488(370)$ & $0,010^{*}$ \\
\hline $\begin{array}{l}\text { Tiempo recolección secreciones (horas) } \\
\text { Media (SD) }\end{array}$ & 42 & $101,4(79,01)$ & $101,3(91)$ & $101,4(75)$ & 0,702 \\
\hline $\begin{array}{l}\text { Relación Peso/Tiempo (gr/h) } \\
\text { Media (SD) }\end{array}$ & 40 & $2,07(2,70)$ & $2,1(4)$ & $2,1(2)$ & 0,312 \\
\hline
\end{tabular}

Tabla 1. Características clínicas de la población estudiada

Tabla 2. Comparación del aspirado del tratamiento estándar vs SUPRAtube.

\section{RESULTADOS}

La aspiración supraglótica o el tratamiento convencional se realizó durante $108 \pm 88 \mathrm{~h}$ continuadas, desde el inicio hasta inmediatamente antes de la extubación. El volumen total aspirado mostró una amplio rango ( 7 hasta $880 \mathrm{ml} / \mathrm{dia}$ ) en los grupos de estudio. En el grupo control, el volumen de aspiración fue de $25 \pm 6 \mathrm{ml} /$ dia mientras que en el grupo SUPRAtube fue de $269 \pm 48 \mathrm{ml} / \mathrm{dia}(p<0,001)$. La relación de peso/tiempo fue de $0,3 \pm 0,1$ $\mathrm{gr} /$ hora en el grupo control, mientras en el grupo SUPRAtube la relación peso/tiempo fue de $3 \pm 1 \mathrm{gr} / \mathrm{h}(\mathrm{p}<0,01)$. El dispositivo SUPRAtube se asoció a mayor resolución de las anormalidades broncoscópicas no-infecciosas ( $11 \mathrm{vs.} 56 \%, p=0,04$ ). Los cultivos aerobios de las secreciones del broncoaspirado se negativizaron en $88 \%$ de los controles vs. $93 \%$ del grupo intervenido ( $p=n s$ ).

\section{CONCLUSIONES}

La aspiración mediante el SUPRAtube en pacientes intubados y ventilados mecánicamente pone en evidencia que el volumen que se acumula en la región supraglótica está muy subestimado en el entorno clínico. El análisis preliminar de este ensayo pone en evidencia que la aspiración supraglótica continua es una estrategia complementaria eficaz para disminuir tanto el volumen de contenido orofaríngeo como las complicaciones infecciosas y no-infecciosas en pacientes intubados y ventilados mecánicamente.

Subvencionado por Proyecto Supranav, COLCIENCIAS 833-2018 Código 65667778334. 